\title{
Stellar Rotation in Kepler: Forward Modeling of the Kepler Period Distribution
}

\author{
Jennifer L. van Saders ${ }^{1,2, a}$, Marc H. Pinsonneault ${ }^{2}$, Rafael A. García ${ }^{3}$, and Tugdual Ceillier ${ }^{3}$ \\ 1 Observatories of the Carnegie Institution for Science, 813 Santa Barbara Street, Pasadena, CA 91101 \\ 2 Department of Astronomy, The Ohio State University, Columbus, OH 43210, USA \\ 3 Laboratoire AIM, CEA/DSM - CNRS - Univ. Paris Diderot - IRFU/SAp, Centre de Saclay, F-91191 \\ Gif-sur-Yvette Cedex, France
}

\begin{abstract}
The Kepler mission has made it possible to detect the signatures of surface rotation in tens of thousands of stars across many different spectral types, ages, and evolutionary states. While it is tempting to use these rotation rates as a means to determine the ages of field stars in Kepler via the gyrochronology relationships, we show that 1) only a fraction of these stars should be viable targets for the existing period-age relationships due to "contamination" from hot stars and subgiants, and 2) that apparent age trends in the rotation distributions can be explained with an activity-based detection bias. We have performed a forward modeling exercise in an effort to reproduce the observed distribution of rotation periods.
\end{abstract}

\section{Rotation in context}

All stars rotate, with rotation periods set by a combination of initial conditions, angular momentum loss, internal angular momentum transport, and the physical expansion/contraction of the star. The decline in the rotation rates of cool main sequence stars as a function of time suggests that rotation might be used as a means of inferring stellar ages through "gyrochronology" [1,2]. Empirical periodage relationships exist, but are only calibrated for the cool dwarfs where strong spin-down due to magnetic braking results in a relationship between period and age for ages $>0.5$ Gyr for sun-like stars $[2,4,5]$.

In the case of the Kepler field, however, we should observe a (biased) mixture of stellar populations, not all of which have gyrochronology relationships. In particular, we expect three distinct populations of rotating stars: the cool dwarfs, which undergo magnetic braking and should be observed as slow rotators, hot stars $\left(T_{e f f}>6250 \mathrm{~K}\right)$, whose convective envelopes are too thin to support magnetic braking and should be rapidly rotating, and subgiants, where the increase in the stellar moment of inertia due to evolution contributes substantially to stellar spin-down [3]. Each of these three groups can overlap in period-space, while having a very different rotational histories. In addition to these population effects, rotation samples are undoubtedly subject detection biases which will tend to disfavor old, quiet, and slowly rotating stars. The use of gyrochronology with Kepler stars will therefore need to account for both population and detection effects before any conclusions about age distributions can be drawn.

\section{Examining Stellar Populations with the Asteroseismic Sample}

To demonstrate the importance of stellar populations in the interpretation of rotation periods, we examine the well-studied subset of stars for which we have asteroseismic detections, with information

a e-mail: jvansaders@obs.carnegiescience.edu

This is an Open Access article distributed under the terms of the Creative Commons Attribution License 4.0, which permits unrestricted use, distribution, and reproduction in any medium, provided the original work is properly cited. 
about the masses, ages, evolutionary states, and rotation periods [6,7]. This sample is shown in Fig 1 and color-coded according to the three stellar populations (cool dwarfs, hot dwarfs, and subgiants). The two curves represent the period-age relationships at two extremes of the stellar mass range over which they are calibrated [4]. All stars obeying a period-age relationship like that of Mamajek \& Hillenbrand 2008 should fall between these two curves, their exact position determined by their mass (and composition).

The sample, taken as a whole, does not appear to obey these period-age relationships, and it is not until we examine the sub-populations that trends become clear. Cool dwarfs, on average, do in fact fall between the curves where we would expect a gyrochronologically viable target to exist. Because hot stars do not undergo magnetic braking, they exist at rotation periods that are too short for their age, and show no discernable period trend with age. Subgiants, on the other hand, are far younger, given their periods, than one would expect from gyrochronology. This is due to the fact that subgiant spin-down is no longer solely dependent on magnetic braking, but is also affected by the substantial increase in the stellar moment of inertia as the star evolves. This subset of well-studied stars serves to demonstrate the mixed population of rotating stars present in the Kepler field, and the dangers of interpreting rotation rates without evolutionary state information.

\section{Forward Modeling a Rotating Stellar Population}

To further examine the importance of stellar populations and selection effects, we design a forward modeling exercise, using surface rotation periods predicted by stellar models and a stellar population model of the Kepler field generated with the TRILEGAL code (model from Mauro Barbieri, private communication) [3,8]. Stellar models assume solid body rotation, and magnetic braking of the form (Pinsonneault et al. 2014, in prep):

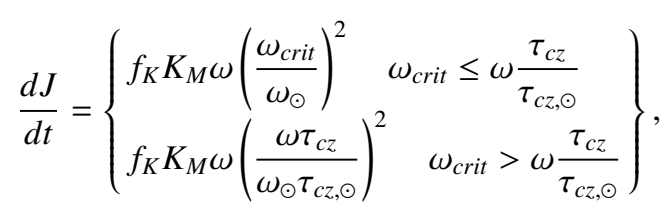

where $\tau_{c z}$ is the convective overturn timescale. Initial rotation rates are calibrated using data from young open clusters. To maintain internal consistency, the masses, ages, and compositions of the TRILEGAL population model are used as inputs to the stellar evolution grids, which then provide rotation periods, effective temperatures, gravities, and all other necessary parameters.

The output of this forward modeling exercise is pictured in a series of panels in Fig. 2. In the first panel we show the observed distribution from McQuillan et al. 2014, which is qualitatively similar to those found by other authors in the literature [9-11]. Below the observed distribution is our modeled population, showing all stars brighter than $K_{p}=16$ and subject to the same $\log g$ and $T_{\text {eff }}$ cuts as those in the McQuillan sample $\left(T_{\text {eff }}<6500 \mathrm{~K}\right.$, and [12] cuts to exclude giants), in addition to a restriction that stars fall within the ranges: $0.4<M_{\odot}<1.7,-0.4<[\mathrm{Fe} / \mathrm{H}]<+0.4$, and $0.5<t<14$ Gyr to fall within the stellar model grid. Stars are color-coded in the same manner as Fig. 1. We see that despite the selections, all three classes of rotators are present, and overlap. Therefore, in practice, unless we have additional information that allows us to determine that a star is a cool dwarf, we cannot reliably determine its age based on rotation in regions where there is heavy overlap among the three populations.

Our initial model predicts far more stars at long periods than are actually observed. This behavior at long periods motivates a final selection criterion: we make the assumption that the activity of stars is correlated with the Rossby number, defined as Ro $=P_{r o t} / \tau_{c z}$, where $\tau_{c z}$ is the convective overturn timescale [13]. Under this assumption, we postulate that there exists some critical Rossby number above which we are no longer able to observe spot modulation, either due to the low amplitude of the variability, or a true lack of variability. In either case, we assume that detectability is related to the Rossby number. Panel 3 shows the period distribution of a magnitude limited sample with Ro $<2$. This simple selection qualitatively reproduces the observed sharp edge at long rotation periods, and 


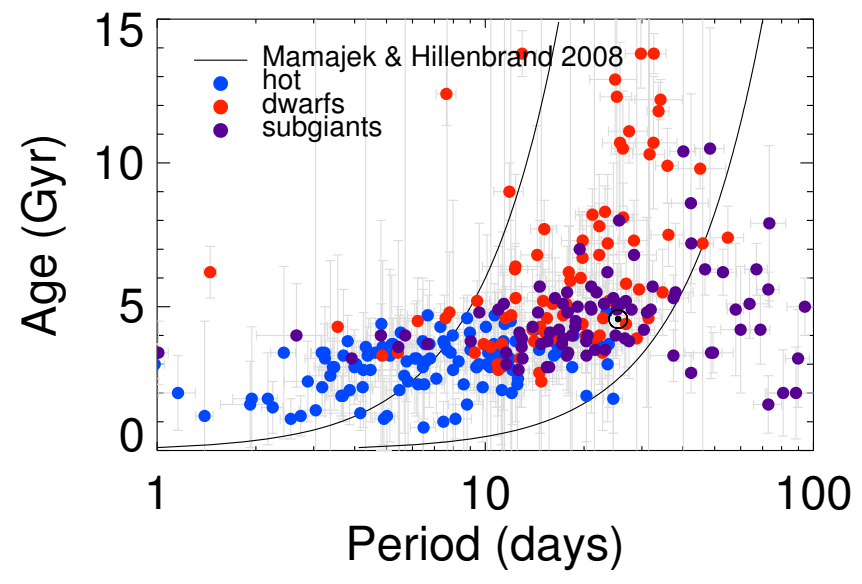

Fig. 1. A subset of asteroseismic targets which emphasizes the mixed nature of stellar populations [7,6]. Cool stars $\left(T_{\text {eff }}<6250 \mathrm{~K}\right)$ are shown in red, hot stars in blue, and subgiants (defined as $\log g<$ $4.0)$ in purple. Gyrochronology relations for $B-V=0.4$ and $B-V=0.9$ from are shown as black curves: stars that obey these gyrochronology relations should fall between these two curves [4].

requires no special age distribution within the stellar population. The bottom panel of Fig 2 shows the age distributions in the samples with and without the Rossby cut: by imposing a maximum observable Rossby number, we limit the age of the stellar population that will actually be visible in spot modulation. Although Ro $=2$ was chosen here to reproduce the location of the cutoff in the period distribution, the shape of "Rossby envelope" comes only from the theoretical predictions for Rossby number as a function of mass and evolutionary state, and has not been fine-tuned.

Notably, a population of subgiants survive this Rossby cut, and are present and detectable at periods longer than those of their main sequence counterparts at a given effective temperature. These subgiants have deeper convective envelopes, longer convective overturn timescales, and therefore lower Rossby numbers at fixed period than main sequence stars at the same effective temperature. Potential subgiants in the McQuillan sample can be seen at long periods at $T_{\text {eff }} \sim 5500 \mathrm{~K}$.

\section{Conclusion}

We must account for stellar populations and selection biases when using rotation periods as a stellar diagnostic: there are many stars that fall within the period ranges for which there exist gyrochronology relationships, and yet do not obey the relations, because the mapping between period and age is different for hot stars and subgiants. We must also be careful to interpret the rotation distribution in the context of selection effects, which can severely bias the set of gyrochronological ages we infer.

In addition to the work presented here, we also caution that blending, whether due to chance alignments of stars in the large Kepler pixels or due to true binaries, can complicate the interpretation of periods. Blends can inhabit the same regions of period-temperature space and masquerade as true sources, when in fact the detected rotation period is not that of the star dominating the light. In addition, synchronized binaries may be responsible for a portion of the rapidly rotating subset of stars, and further complicate the interpretation of rotation periods. A full quantification of the impact of blending is left to later work.

\section{References}

1. Skumanich, A. 1972, ApJ, 171, 565

2. Barnes, S. A. 2007, ApJ, 669, 1167

3. van Saders, J. L., \& Pinsonneault, M. H. 2013, ApJ, 776, 67

4. Mamajek, E. E., \& Hillenbrand, L. A. 2008, ApJ, 687, 1264 

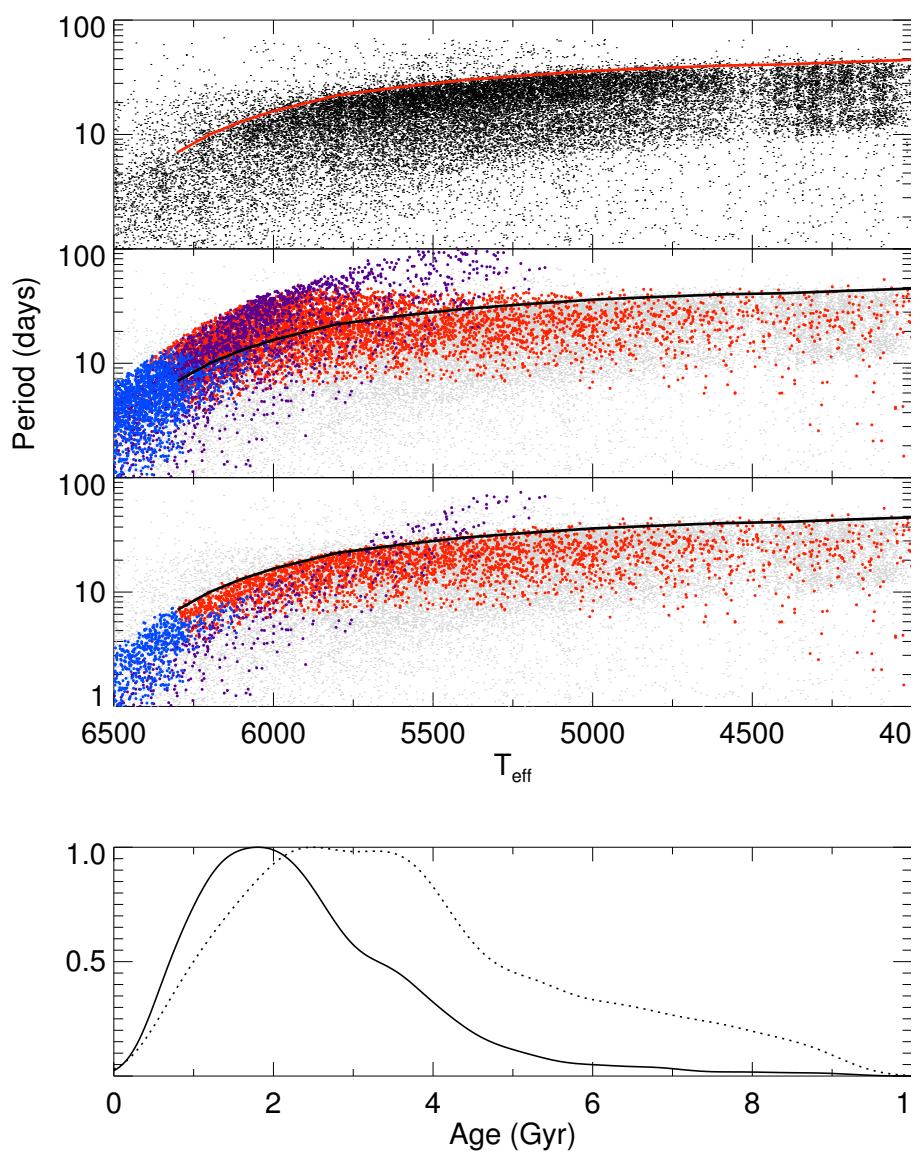

Fig. 2. Top panel: Data from [9], reproduced here. We mark, as a red curve, the observed edge to the period distribution to guide the eye. Second panel: Our population model, with dwarfs in red, hot stars in blue, and subgiants in purple. The McQuillan et al. sample is plotted in light gray points, and the curve marking the edge in the period distribution from the first panel reproduced in black. Third panel: The population model, but with a Rossby cut of Ro $=2.0$ applied. Bottom panel: The age distributions of the samples in panels 2 and 3: the dotted line represents the full population, and the solid line the same population subject to a cut in Rossby number. (Please note that each curve has been arbitrarily normalized for display.)

5. Epstein, C. R., \& Pinsonneault, M. H. 2014, ApJ, 780, 159

6. Chaplin, W. J., et al. 2014, ApJS, 210, 1

7. Garcia, R. A., et al. 2014, A\&A, 572, 34

8. Girardi, L., Groenewegen, M. A. T., Hatziminaoglou, E., \& da Costa, L. 2005, A\&A, 436, 895

9. McQuillan, A., Mazeh, T., \& Aigrain, S. 2014, ApJS, 211, 24

10. Nielsen, M. B., Gizon, L., Schunker, H., \& Karoff, C. 2013, A\&A, 557, L10

11. Reinhold, T., Reiners, A., \& Basri, G. 2013, A\&A, 560, A4

12. Ciardi, D. R., et al. 2011, AJ, 141, 108

13. Noyes, R., et al. 1984, ApJ, 279, 763 